\title{
Circadian and Sleep Dysfunctions in Neurodegenerative Disorders-An Update
}

\author{
Karim Fifel ${ }^{1 * \dagger}$ and Aleksandar Videnovic ${ }^{2 \dagger}$ \\ ${ }^{1}$ International Institute for Integrative Sleep Medicine (WPI-IIIS), University of Tsukuba, Tsukuba, Japan, ${ }^{2}$ Movement \\ Disorders Unit and Division of Sleep Medicine, Massachusetts General Hospital and Harvard Medical School, Boston, MA, \\ United States
}

OPEN ACCESS

Edited by:

Christopher S. Colwell,

University of California, Los Angeles,

United States

Reviewed by:

Erik Steven Musiek,

Saint Louis University, United States

Michelangelo Maestri,

Università degli Studi di Pisa, Italy

${ }^{*}$ Correspondence:

Karim Fifel

fifel-k@hotmail.com

orcid.org/0000-0001-6259-9336

tThese authors have contributed

equally to this work

Specialty section:

This article was submitted to

Sleep and Circadian Rhythms,

a section of the journal

Frontiers in Neuroscience

Received: 09 November 2020

Accepted: 29 December 2020

Published: 18 January 2021

Citation:

Fifel K and Videnovic A (2021)

Circadian and Sleep Dysfunctions

in Neurodegenerative Disorders - An

Update. Front. Neurosci. 14:627330.

doi: 10.3389/fnins.2020.627330
Disruptions of sleep and circadian rhythms are among the most debilitating symptoms in patients with neurodegenerative diseases. Their underlying pathophysiology is multilayered and multifactorial. Recent evidence suggests that sleep and circadian disturbances may influence the neurodegenerative processes as well as be their consequence. In this perspective, we provide an update of the current understanding of sleep and circadian dysregulation in Alzheimer's, Parkinson's, and Huntington's diseases.

Keywords: Alzheimer, Parkinson, Huntington, sleep, circadian system

\section{INTRODUCTION}

Neurodegenerative disorders are characterized by a constellation of multiple impairments in diverse functional domains (Jucker and Walker, 2013). In addition to the primary symptoms characterizing each neurodegenerative disease [i.e., memory dysfunction in Alzheimer's disease (AD) and motor disorders in Parkinson's disease (PD), and Huntington's disease (HD)], several physiological functions deteriorate over disease progression and will ultimately dominate the clinical picture at advanced stages of the diseases (Bates et al., 2015; Masters et al., 2015; Poewe et al., 2017; Long and Holtzman, 2019). The primary neurodegenerative processes affect regions involved in the regulation of sleep, alertness and circadian rhythms, leading to disrupted sleep-wake cycles and circadian dysregulation (Coogan et al., 2013; Videnovic and Golombek, 2013, 2017; Videnovic et al., 2014a,b; Mattis and Sehgal, 2016; Musiek and Holtzman, 2016; Videnovic and Willis, 2016; De Pablo-Fernández et al., 2017; Fifel, 2017; La Morgia et al., 2017; Li et al., 2017; De Lazzari et al., 2018; Leng et al., 2019; Duncan, 2020). Increasing evidence points to deleterious effects of disrupted sleep and circadian homeostasis on the biological processes that underlie neurodegeneration. This bi-directional relationship between neurodegeneration and sleep and circadian systems opens immense opportunities for the development of novel treatment approaches for these disorders (Musiek and Holtzman, 2016; Leng et al., 2019). In this perspective, we summarize the symptomatology of sleep and circadian rhythms in neurodegenerative disorders, with an emphasis on the most common neurodegenerative disorders-AD, PD, and HD. Mechanistic insights mediating sleep and circadian dysfunction in these neurodegenerative diseases are highlighted as 
well. We have focused on human clinical investigations with occasional referral to animal studies for mechanistic insights.

\section{CIRCADIAN DYSFUNCTION IN NEURODEGENERATIVE DISORDERS}

Circadian organization of biological processes is manifest, and plays a paramount role, at every level of organization from gene expression and cellular redox state regulation to intraand inter-organ physiological coordination (Bass and Lazar, 2016; Patke et al., 2020). Given this complex and hierarchical organization of circadian biology, it is not a surprise that chronic breakdown of circadian rhythms is a significant risk factor for a range of diseases, including cancer, metabolic disorders, psychiatric disturbances, and neurodegenerative diseases (Bass and Lazar, 2016; Patke et al., 2020). In neurodegenerative disorders, three types of circadian alterations can be defined: behavioral, physiological and molecular alterations.

The most evident and extensively studied overt behavioral circadian disturbance in patients with neurodegenerative diseases is the alteration of sleep/wake behavior. Given the complex and widespread neuronal centers governing the quality and quantity of sleep/wake cycle (Saper et al., 2005) and the widespread nature of neurodegeneration and neural dysfunction in patients with neurodegenerative disorders (Jucker and Walker, 2013), sleep disruption usually precedes respective clinical diagnoses by several years and contribute significantly to the deterioration of the patients' quality of life. Some of the sleep alterations in neurodegenerative diseases are exaggerated forms of age-related sleep alterations (Mander et al., 2017). A typical example of this in $\mathrm{AD}, \mathrm{PD}$, and $\mathrm{HD}$ patients is the progressive fragmentation of sleep cycle over time. A more detailed discussion of sleep disorders in $\mathrm{AD}, \mathrm{PD}$, and $\mathrm{HD}$ diseases will follow in the next section. Other studies have capitalized on actigraphy to continuously monitor the progression of rest/activity rhythms in community-dwelling patients with neurodegenerative diseases (Leng et al., 2019). These studies have shown several alterations including frequent daytime naps, altered phase angles as evident by shifts in both bedtime and wake times, rest/activity fragmentation, and reduced amplitudes (Ortiz-Tudela et al., 2014; Hooghiemstra et al., 2015; Wang et al., 2015; Weissova et al., 2016). Interestingly, four recent studies have revealed robust association between weakened rest/activity rhythmicity and the subsequent development of $\mathrm{AD}$ and $\mathrm{PD}$ years later (Musiek et al., 2018; Lysen et al., 2019; Leng et al., 2020; Li et al., 2020). Circadian disturbances could therefore act as independent risk factor for the development of neurodegenerative diseases. Future studies should investigate the mechanisms behind these associations. Further, circadian rhythms could be a promising therapeutic target for the prevention and management of neurodegenerative diseases.

Alterations of additional physiological processes that are under the regulation of the circadian pacemaker, the suprachiasmatic nucleus $(\mathrm{SCN})$ have been reported in these neurodegenerative disorders (Fifel, 2017; Fifel and DeBoer, 2020; Fifel and Videnovic, 2020). Several investigations in AD, PD and
HD patients have revealed alterations of the circadian amplitude of cortisol rhythm (Hartmann et al., 1997; Hatfield et al., 2004; Aziz et al., 2009a; Adamczak-Ratajczak et al., 2017), reversal or complete loss of nychthemeral modulation of blood pressure and heart rate variability (Ejaz et al., 2006; Chen et al., 2013; Vetrano et al., 2015; Diago et al., 2018; Baschieri and Cortelli, 2019), phase desynchrony and amplitude disruption of melatonin-release cycle (Skene and Swaab, 2003; Aziz et al., 2009b; Kalliolia et al., 2014; Adamczak-Ratajczak et al., 2017; Fifel, 2017), dampening or reversal of the diurnal pattern of urine excretion (Hineno et al., 1994; Ouslander et al., 1998) and impaired body temperature (Raupach et al., 2019). Collectively these alterations demonstrate that patients with neurodegenerative diseases exhibit a global circadian desynchrony. This state of systemic desynchrony is known to precipitate deleterious impact on global health that is worse than having a dysfunctional SCN clock (Fernandez et al., 2014) and is therefore expected to causally contribute to health impairment in patients with neurodegenerative diseases.

The additional body of evidence linking circadian disturbances with neurodegenerative disorders comes from studies investigating circadian molecular markers in patients with neurodegenerative diseases. In PD patients, although all clock genes were not affected, significant alterations of the timing and amplitude of core clock genes such as Bmall, Bmal2, Per2, and Rev-erb $\alpha$ were reported (Cai et al., 2010; Breen et al., 2014). Breen et al. reported a loss of the circadian expression of Bmall in peripheral mononuclear cells of PD patients (Breen et al., 2014). Few studies have also examined the contribution of circadian dysfunction to health impairments in PD patients by exploring the associations between singlenucleotide polymorphism of several clock-related genes and motor and non-motor symptoms of PD (Ding et al., 2011; Hua et al., 2012; Gu et al., 2015; Lou et al., 2018; Mao et al., 2018). Polymorphisms in clock-related genes such as ARNTL, thyrotroph embryonic factor gene, Clock, were proposed as independent risk factors for motor, depression-related and sleep disorders in PD (Gu et al., 2015). These studies suggest that the molecular mechanisms of the clock in peripheral organs of PD patients are altered and could contribute to the behavioral and physiological desynchronization observed in patients with PD (Fifel, 2017). Similar studies centered on clock genes expression in $\mathrm{AD}$ and $\mathrm{HD}$ are scant. However, few pathological studies using post-mortem brain tissue have shown a loss of the diurnal profile of clock genes (Bmal1, Cry1, Per1) in the pineal gland of patients with preclinical and manifest $\mathrm{AD}$ (Wu et al., 2006). Similarly, by examining the expression of clock genes per1, per2 and bmall in several brain structures, Cermakian et al. (2011) reported a dyssynchronous expression of several clock genes in different brain structures. In addition to altered clock gene expression, the expression of key SCN neuropeptides such as vasopressin, AVP and VIP, is significantly decreased in patients with AD and HD (Zhou et al., 1995; Liu et al., 2000; Wu et al., 2007; van Wamelen et al., 2013). Collectively, the behavioral, physiological and molecular alterations of the circadian system strongly implicate their involvement as a significant causal factor in the impairment of the quality of life in patients with neurodegenerative diseases. 
Similar to the underlying mechanisms of sleep disturbances, the mechanisms of circadian alterations are also multifactorial involving the interactions between several intrinsic neurodegenerative processes (i.e., inflammation, oxidative stress) as well as a dysfunctional communication between central and peripheral clocks (Musiek and Holtzman, 2016; Chauhan et al., 2017). Recently, several molecular pathways linking multiple neurodegenerative processes with the genetic machinery of the circadian clock have been identified (Musiek and Holtzman, 2016; Sharma et al., 2020). However, translational insights from these mechanistic studies aimed at restoring a robust, yet flexible, circadian rhythms in patients with neurodegenerative disorders have not yet materialized.

From a neural network perspective, circadian rhythms are generated by a well-coordinated communication between peripheral clocks and the central clock in the SCN. This is achieved by both neural connections and humoral signals (Hastings et al., 2003; Bass and Lazar, 2016). In neurodegenerative diseases, significant impairments in different parts of this circadian neuronal network have been documented and have been recently reviewed elsewhere (Chauhan et al., 2017; Fifel, 2017; Fifel and DeBoer, 2020; Fifel and Videnovic, 2020). This knowledge is of a paramount importance for future attempts to develop specific network-based therapies for circadian and sleep symptoms in neurodegenerative diseases.

\section{SLEEP DYSFUNCTION IN NEURODEGENERATIVE DISORDERS}

Disturbances of sleep and alertness are common in neurodegenerative disorders such as Alzheimer's, Parkinson's, and Huntington's diseases. Despite its common presence, sleep disruption is frequently under-reported by patients and their caregivers and under-diagnosed by healthcare professionals. Disrupted sleep negatively affects the quality of life and may impact the safety of patients. Increasingly manifested as these disorders progress, sleep dysfunctions may also predate their onset by years, even decades. For example, REM Sleep Behavior Disorder represents the most significant prodromal manifestation of a synuclein-specific neurodegenerative disorder, and excessive daytime sleepiness has been linked with the development of Parkinson's disease (Abbott et al., 2005; Hogl et al., 2018).

\section{Alzheimer's Disease}

Alzheimer's disease (AD), the most common neurodegenerative disorder, has been associated with sleep and circadian disturbances for a long time. Recent evidence strongly suggests a bi-directional relationship between $\mathrm{AD}$ and sleep/circadian disruption (Wang and Holtzman, 2020). Sleep changes that accompany aging are accentuated among individuals affected by $\mathrm{AD}$. In $\mathrm{AD}$, sleep becomes fragmented leading to shorter total sleep time, excessive sleepiness may develop with subsequent frequent napping, and sundowning is often associated with behavioral changes and agitation. These changes affect not only patients but may cause a significant strain on their caregivers
(Terum et al., 2017). Alterations in sleep architecture in AD encompass less frequent and poorly formed spindles and $\mathrm{K}$ complexes, reduced slow-wave sleep, and changes in the spectral properties of REM sleep (Vitiello et al., 1990; Petit et al., 2004; Guarnieri et al., 2020). Recently the contribution of Obstructive sleep apnea to cognitive impairments in $\mathrm{AD}$ patients has been recognized (Bubu et al., 2020; Liguori et al., 2020). Burgeoning evidence demonstrates an increased risk of developing $\mathrm{AD}$ in individuals with a long-standing history of disturbed sleep (Virta et al., 2013; Pase et al., 2017). Several recently published meta-analyses reported that individuals with sleep problems had up to 4 times higher risk of $\mathrm{AD}$, cognitive impairment, and preclinical AD (Bubu et al., 2017; Shi et al., 2018). Advance in biomarkers of AD have allowed more sophisticated analysis of the relationship between sleep and AD. Disruption of slow-wave sleep is associated with increased levels of beta-amyloid, whose deposition may impair slow-wave sleep (Ju et al., 2017). Tau aggregation and spreading is known to drive AD neurodegeneration (Holth et al., 2019; Long and Holtzman, 2019). Recent investigations both in mice and humans have shown that not only beta-amyloid, but also tau aggregation is regulated by sleep/wake cycle (Holth et al., 2019). More specifically, recent longitudinal studies have identified changes in NREM slow-wave activity, especially at $1-2 \mathrm{~Hz}$, as specific and reliable EEG markers in forecasting the rate of $A \beta$ accumulation over several subsequent years (Lucey et al., 2019; Winer et al., 2020). Changes in REM sleep, such as reduced REM sleep and prolonged REM latency have also been linked with the development of dementia (Pase et al., 2017). Another mechanism implicating sleep in the progression of AD pathology is related to the glymphatic system. This system, which clears the brain of protein waste products, is mostly active during sleep and suppressed during wakefulness (Xie et al., 2013). Clearance of amyloid or tau proteins by the glymphatic system may therefore be reduced on a background of disrupted sleep (Rasmussen et al., 2018; Nedergaard and Goldman, 2020).

Proper assessment of disturbed sleep in $\mathrm{AD}$ necessitate that caregivers be interviewed, as patients may not be aware of these sleep disturbances. From an evidence-based perspective, employing good sleep hygiene, and trazodone seem beneficial for improving sleep quality (McCleery et al., 2014). Trazodone may exhibit additional benefits on cognition with long-term use (reviewed in Ferini-Strambi et al., 2020). Several studies that examined the role of melatonin in managing poor sleep associated with $\mathrm{AD}$ found no beneficial effects of several sleep metrics (McCleery et al., 2014).

\section{Parkinson's Disease}

James Parkinson reported on disrupted sleep in his initial clinical depiction of PD in 1817. The impact of disturbed sleep and alertness, however, has only been systematically examined over the past several decades. Excessive daytime sleepiness and/or disrupted nighttime sleep affect the majority of patients during the lifetime of their PD (Chahine et al., 2017). Fragmented sleep is the most prevalent sleep problem in the PD population (Chahine et al., 2017). Up to $80 \%$ of PD patients report poor sleep, which has been characterized by lower sleep times and 
sleep efficiency on polysomnography (Yong et al., 2011). The multifactorial etiology of poor sleep encompasses co-existent sleep and psychiatric disorders, overnight re-emergence of PD symptoms, effects of antiparkinsonian medications on sleep, autonomic dysfunction as well as neurodegeneration of brain regions responsible for the regulation of the sleep-wake cycle.

Primary sleep disorders have some unique features when co-expressed with PD. REM Sleep Behavior Disorder (RBD), a parasomnia characterized by loss of muscle atonia during REM sleep and dream enactment behavior, affects up to $60 \%$ of patients with PD (Sixel-Doring et al., 2016). RBD is of special significance for $\mathrm{PD}$ as it represents the most significant prodromal manifestation of an evolving PD and other synucleinopathies-dementia with Lewy bodies and multiple system atrophy. Dream enactment behaviors of RBD pose a substantial risk for injuries of patients and their bed partners. Restless Legs Syndrome (RLS) is another primary sleep disorder frequently associated with PD. In contrast to RBD, whether RLS is also a risk factor for subsequent development of $\mathrm{PD}$ is still a subject of debate (Alonso-Navarro et al., 2019; You et al., 2019). True prevalence of RLS in PD is not well established as many mimickers of RLS frequently affect PD patients, such as akathisia, rigidity, and leg cramps (You et al., 2019). While both RLS and PD respond favorably to dopaminergic medications and are worsened by the administration of dopamine blocking medications, there are sharp differences from a neuropathological standpoint. Dopaminergic neuronal loss with co-existent increased iron in substantia nigra PD is in contrast to the absence of neuronal loss and reduced iron content in RLS (Ferini-Strambi et al., 2018). Initially reported within the context of parkinsonism during the 1918 Spanish flu pandemic, sleep disordered breathing seem to have similar prevalence in the PD and general populations (Cochen De Cock et al., 2010). Important differentiators include a higher proportion of central and mixed apneas among PD patients, and a lack of strong associations between elevated body mass index and sleep disordered breathing in the PD population (Lajoie et al., 2020). Excessive daytime sleepiness (EDS) became a focus of medical attention in early the 1990s when reports of EDS and "sleep attacks" in association with the use of dopamine agonists were published (Frucht et al., 1999). The prevalence of EDS in pivotal clinical trials of major dopaminergic medications was 15-30\%; Treatment with dopamine agonists carries the highest risk of EDS, followed by the combination therapy with levodopa and dopamine agonist, and levodopa monotherapy (extensively reviewed by Chahine et al., 2017). EDS is more prevalent in PD compared with other chronic diseases (Tandberg et al., 1999). While poor alertness and disturbed sleep share causative factors, there are unique mechanisms underlying disturbed alertness in PD. These include loss of hypocretin neurons in the hypothalamus, neurodegeneration of wake-promoting centers and its projections within the brainstem, and circadian disruption discussed in other segments of this article (Fronczek et al., 2007; Fifel and Videnovic, 2020).

In summary, the landscape of disturbed sleep and alertness in PD is quite rich. The diagnosis and treatment approaches for disturbances of sleep and wake cycles have been presented in several well-written reviews (Amara et al., 2017; Loddo et al., 2017). It is worth emphasizing that further work is needed to develop new and optimize existing PD-specific diagnostic tools for disorders of sleep and wake. There is an overwhelming lack of effective, evidence-based treatments of sleep dysfunction in PD (Seppi et al., 2019). This dictates the need for clinical investigations of sleep therapeutics in PD. Sleep may be an important therapeutic target for alleviating both motor and non-motor manifestations of PD. These approaches may also have favorable effects on the disease progression itself.

\section{Huntington's Disease}

Huntington's disease (HD) is a progressive neurodegenerative disease characterized by movement deficits, cognitive deterioration, and psychiatric symptoms. Sleep disturbances are common in HD and affect up to $90 \%$ of patients. Despite its common occurrence in $\mathrm{HD}$, sleep disorders have not been so thoroughly investigated as in other neurodegenerative disorders (Zhang et al., 2019). Although disturbed sleep in HD correlated with its severity and duration of illness, poor sleep has been reported in the early stages of the disease, even among asymptomatic carriers of the HD mutation (Arnulf et al., 2008; Lazar et al., 2015). Polysomnography in HD reveals fragmented sleep with reduced N3 stage and increased sleep spindle density (Wiegand et al., 1991). REM sleep appears to be reduced in $\mathrm{HD}$, although this finding has been not replicated in all studies (Hansotia et al., 1985; Wiegand et al., 1991). RBD has been reported in HD (Videnovic et al., 2009). Poor sleep may be associated with depression and other neuropsychiatric manifestations of the disease (Videnovic et al., 2009). This emphasizes the need for timely diagnosis and aggressive treatment of disturbed sleep in patients with HD.

Diagnosis of disorders of sleep and wake in the HD population relies on instruments and tests used in general sleep medicine. Complexities of HD that influence sleep patterns in this population necessitate the development of novel assessment methods for sleep and alertness in HD. There is a paucity of therapeutic studies that focused on disturbed sleep in HD. Managing sleep disfunction is further complicated by complex medication regimens that include medications that may negatively impact sleep and/or daytime alertness. Nonpharmacological strategies such as physical exercise may be a promising approach in the management of sleep dysfunction in HD (Mueller et al., 2019).

\section{CONCLUSION AND PERSPECTIVES}

Sleep and circadian rhythms are interconnected and allencompassing physiological systems that affect virtually all biological functions. Unsurprisingly, circadian disruption can precipitate considerable impairments on human health. In neurogenerative diseases, although it is well established that sleep and circadian dysfunctions are both a consequence of and a causal factor of neurodegenerative processes, holistic 
investigation of clocks, sleep and neurodegeneration is still in its infancy. The extent of neuropathological dysfunctions in sleep and circadian neural centers is still not fully understood. Additionally, the exact role of clock genes in the regulation of cellular function in different brain structures and peripheral organs is unknown. The accelerating progress in this field is holding promising insights into the development of efficient therapies for improving health condition in patients with neurodegenerative diseases. Chronotherapies such as the use of timed bright light therapy is a recent example of how fundamental research in this field have yielded positive outcomes in patients with neurodegenerative disorders (Fifel and Videnovic, 2018, 2019).

\section{REFERENCES}

Abbott, R. D., Ross, G. W., White, L. R., Tanner, C. M., Masaki, K. H., Nelson, J. S., et al. (2005). Excessive daytime sleepiness and subsequent development of Parkinson disease. Neurology 65, 1442-1446.

Adamczak-Ratajczak, A., Kupsz, J., Owecki, M., Zielonka, D., Sowinska, A., Checinska-Maciejewska, Z., et al. (2017). Circadian rhythms of melatonin and cortisol in manifest Huntington's disease and in acute cortical ischemic stroke. J. Physiol. Pharmacol. 68, 539-546.

Alonso-Navarro, H., García-Martín, E., Agúndez, J. A. G., and Jiménez-Jiménez, F. J. (2019). Association between restless legs syndrome and other movement disorders. Neurology 92, 948-964. doi: 10.1212/wnl.0000000000007500

Amara, A. W., Chahine, L. M., and Videnovic, A. (2017). Treatment of sleep dysfunction in Parkinson's Disease. Curr Treat Options Neurol. 19:26.

Arnulf, I., Nielsen, J., and Lohmann, E. (2008). Rapid eye movement sleep disturbances in Huntington disease. Arch. Neurol. 65, 482-488.

Aziz, N. A., Pijl, H., Frölich, M., van der Graaf, A. W., Roelfsema, F., and Roos, R. A. (2009a). Increased hypothalamic-pituitary-adrenal axis activity in Huntington's disease. J. Clin. Endocrinol. Metab. 94, 1223-1228.

Aziz, N. A., Pijl, H., Frölich, M., Schröder-van der Elst, J. P., van der Bent, C., Roelfsema, F., et al. (2009b). Delayed onset of the diurnal melatonin rise in patients with Huntington's disease. J. Neurol. 256, 1961-1965. doi: 10.1007/ s00415-009-5196-1

Baschieri, F., and Cortelli, P. (2019). Circadian rhythms of cardiovascular autonomic function: physiology and clinical implications in neurodegenerative diseases. Auton. Neurosci. 217, 91-101. doi: 10.1016/j.autneu.2019.01.009

Bass, J., and Lazar, M. A. (2016). Circadian time signatures of fitness and disease. Science 354, 994-999. doi: 10.1126/science.aah4965

Bates, G. P., Dorsey, R., Gusella, J. F., Hayden, M. R., Kay, C., Leavitt, B. R., et al. (2015). Huntington disease. Nat. Rev. Dis. Primers 23:15005.

Breen, D. P., Vuono, R., Nawarathna, U., Fisher, K., Shneerson, J. M., Reddy, A. B., et al. (2014). Sleep and circadian rhythm regulation in early Parkinson disease. JAMA Neurol. 71, 589-595. doi: 10.1001/jamaneurol.2014.65

Bubu, O. M., Andrade, A. G., Umasabor-Bubu, O. Q., Hogan, M. M., Turner, A. D., de Leon, M. J., et al. (2020). Obstructive sleep apnea, cognition and Alzheimer's disease: a systematic review integrating three decades of multidisciplinary research. Sleep Med Rev. 50:101250. doi: 10.1016/j.smrv.2019.101250

Bubu, O. M., Brannick, M., and Mortimer, J. (2017). Sleep, Cognitive impairment, and Alzheimer's disease: a systematic review and meta-analysis. Sleep 40:zsw032.

Cai, Y., Liu, S., Sothern, R. B., Xu, S., and Chan, P. (2010). Expression of clock genes Per1 and Bmal1 in total leukocytes in health and Parkinson's disease. Eur. J. Neurol. 17, 550-554. doi: 10.1111/j.1468-1331.2009.02848.x

Cermakian, N., Lamont, E. W., Boudreau, P., and Boivin, D. B. (2011). Circadian clock gene expression in brain regions of Alzheimer 's disease patients and control subjects. J. Biol. Rhythms 26, 160-170. doi: 10.1177/0748730410395732

Chahine, L. M., Amara, A. W., and Videnovic, A. (2017). A systematic review of the literature on disorders of sleep and wakefulness in Parkinson's disease from 2005 to 2015. Sleep Med Rev. 35, 33-50. doi: 10.1016/j.smrv.2016.08.001

Chauhan, R., Chen, K. F., Kent, B. A., and Crowther, D. C. (2017). Central and peripheral circadian clocks and their role in Alzheimer's disease. Dis. Model. Mech. 10, 1187-1199. doi: 10.1242/dmm.030627

\section{AUTHOR CONTRIBUTIONS}

Both authors listed have made a substantial, direct and intellectual contribution to the work, and approved it for publication.

\section{FUNDING}

KF gratefully acknowledges support during the writing of this article from the TAKEDA foundation. AV received research grant support from the National Institutes of Health (R01NS099055 and R21NS108022).

Chen, H. F., Chang-Quan, H., You, C., Wang, Z. R., Hui, W., Liu, Q. X., et al. (2013). The circadian rhythm of arterial blood pressure in Alzheimer disease (AD) patients without hypertension. Blood Press 22, 101-105. doi: 10.3109/ 08037051.2012.733508

Cochen De Cock, V., Abouda, M., and Leu, S. (2010). Is obstructive sleep apnea a problem in Parkinson's disease? Sleep Med. 11, 247-252.

Coogan, A. N., Schutová, B., Husung, S., Furczyk, K., Baune, B. T., Kropp, P., et al. (2013). The circadian system in Alzheimer's disease: disturbances, mechanisms, and opportunities. Biol. Psychiatry 74, 333-339. doi: 10.1016/j.biopsych.2012. 11.021

De Lazzari, F., Bisaglia, M., Zordan, M. A., and Sandrelli, F. (2018). Circadian rhythm abnormalities in Parkinson's disease from humans to flies and back. Int. J. Mol. Sci. 19:3911. doi: 10.3390/ijms19123911

De Pablo-Fernández, E., Breen, D. P., Bouloux, P. M., Barker, R. A., Foltynie, T., and Warner, T. T. (2017). Neuroendocrine abnormalities in Parkinson's disease. J. Neurol. Neurosurg. Psychiatry 88, 176-185.

Diago, E. B., Martínez-Horta, S., Lasaosa, S. S., Alebesque, A. V., Pérez-Pérez, J., Kulisevsky, J., et al. (2018). Circadian rhythm, cognition, and mood disorders in Huntington's Disease. J. Huntingtons Dis. 7, 193-198. doi: 10.3233/jhd-18029 1

Ding, H., Liu, S., Yuan, Y., Lin, Q., Chan, P., and Cai, Y. (2011). Decreased expression of Bmal2 in patients with Parkinson's disease. Neurosci. Lett. 499, 186-188. doi: 10.1016/j.neulet.2011.05.058

Duncan, M. J. (2020). Interacting influences of aging and Alzheimer's disease on circadian rhythms. Eur. J. Neurosci. 51, 310-325. doi: 10.1111/ejn.14358

Ejaz, A. A., Sekhon, I. S., and Munjal, S. (2006). Characteristic findings on 24-h ambulatory blood pressure monitoring in a series of patients with Parkinson's disease. Eur. J. Intern. Med. 17, 417-420.

Ferini-Strambi, L., Carli, G., Casoni, F., and Galbiati, A. (2018). Restless legs syndrome and Parkinson Disease: a causal relationship between the two disorders? Front. Neurol. 9:551. doi: 10.3389/fneur.2018.00551

Ferini-Strambi, L., Galbiati, A., Casoni, F., and Salsone, M. (2020). Therapy for insomnia and circadian rhythm disorder in Alzheimer Disease. Curr. Treat. Opt. Neurol. 22:4.

Fernandez, F., Lu, D., Ha, P., Costacurta, P., Chavez, R., Heller, H. C., et al. (2014). Circadian rhythm. Dysrhythmia in the suprachiasmatic nucleus inhibits memory processing. Science 346, 854-857. doi: 10.1126/science.1259652

Fifel, K. (2017). Alterations of the circadian system in Parkinson's disease patients. Mov. Disord. 32, 682-692. doi: 10.1002/mds.26865

Fifel, K., and Videnovic, A. (2018). Light therapy in Parkinson's Disease: towards mechanism-based protocols. Trends Neurosci. 41, 252-254. doi: 10.1016/j.tins. 2018.03.002

Fifel, K., and Videnovic, A. (2019). Chronotherapies for Parkinson's disease. Prog. Neurobiol. 174, 16-27. doi: 10.1016/j.pneurobio.2019.01.002

Fifel, K., and Videnovic, A. (2020). Circadian alterations in patients with neurodegenerative diseases: neuropathological basis of underlying network mechanisms. Neurobiol. Dis. 144:105029. doi: 10.1016/j.nbd.2020.105029

Fifel, K., and DeBoer, T. (2020). The circadian system in Parkinson's Disease, multiple system atrophy and progressive supranuclear palsy. Handb Clin Neurol. (in press).

Fronczek, R., Overeem, S., and Lee, S. Y. (2007). Hypocretin (orexin) loss in Parkinson's disease. Brain 130(Pt 6), 1577-1585. 
Frucht, S., Rogers, J. D., Greene, P. E., Gordon, M. F., and Fahn, S. (1999). Falling asleep at the wheel: motor vehicle mishaps in persons taking pramipexole and ropinirole. Neurology 52, 1908-1910. doi: 10.1212/wnl.52.9.1908

Gu, Z., Wang, B., Zhang, Y. B., Ding, H., Zhang, Y., Yu, J., et al. (2015). Association of ARNTL and PER1 genes with Parkinson's disease: a case-control study of Han Chinese. Sci. Rep. 28:15891.

Guarnieri, B., Maestri, M., Cucchiara, F., Lo Gerfo, A., Schirru, A., Arnaldi, D., et al. (2020). Multicenter study on sleep and circadian alterations as objective markers of mild cognitive impairment and Alzheimer's Disease reveals sex differences. J Alzheimers Dis. 78, 1707-1719. doi: 10.3233/jad-200632

Hansotia, P., Wall, R., and Berendes, J. (1985). Sleep disturbances and severity of Huntington's disease. Neurology 35, 1672-1674. doi: 10.1212/wnl.35.11.1672

Hartmann, A., Veldhuis, J. D., Deuschle, M., Standhardt, H., and Heuser, I. (1997). Twenty-four hour cortisol release profiles in patients with Alzheimer's and Parkinson's Disease compared to normal controls: ultradian secretory pulsatility and diurnal variation. Neurobiol. Aging 18, 285-289. doi: 10.1016/ s0197-4580(97)80309-0

Hastings, M. H., Reddy, A. B., and Maywood, E. S. (2003). A clockwork web: circadian timing in brain and periphery, in health and disease. Nat. Rev. Neurosci. 4, 649-661. doi: 10.1038/nrn1177

Hatfield, C. F., Herbert, J., van Someren, E. J., Hodges, J. R., and Hastings, M. H. (2004). Disrupted daily activity/rest cycles in relation to daily cortisol rhythms of home-dwelling patients with early Alzheimer's dementia. Brain 127, 1061-1074. doi: 10.1093/brain/awh129

Hineno, T., Mizobuchi, M., Nishimatsu, O., Horiguchi, J., and Kakimoto, Y. (1994). Day-night variation of urine volume in Parkinson's disease. Jpn. J. Psychiatry Neurol. 48, 583-587. doi: 10.1111/j.1440-1819.1994.tb03018.x

Hogl, B., Stefani, A., and Videnovic, A. (2018). Idiopathic REM sleep behaviour disorder and neurodegeneration - an update. Nat. Rev. Neurol. 14, 40-55. doi: 10.1038/nrneurol.2017.157

Holth, J. K., Fritschi, S. K., Wang, C., Pedersen, N. P., Cirrito, J. R., Mahan, T. E., et al. (2019). The sleep-wake cycle regulates brain interstitial fluid tau in mice and CSF tau in humans. Science 363, 880-884. doi: 10.1126/science.aav2546

Hooghiemstra, A. M., Eggermont, L. H., Scheltens, P., van der Flier, W. M., and Scherder, E. J. (2015). The rest-activity rhythm and physical activity in earlyonset dementia. Alzheimer Dis. Assoc. Disord. 29, 45-49. doi: 10.1097/wad. 0000000000000037

Hua, P., Liu, W., and Kuo, S. H. (2012). Association of Tef polymorphism with depression in Parkinson disease. Mov. Disord. 27, 1694-1697. doi: 10.1002/ mds. 25195

Ju, Y. S., Ooms, S. J., and Sutphen, C. (2017). Slow wave sleep disruption increases cerebrospinal fluid amyloid-beta levels. Brain 140, 2104-2111. doi: 10.1093/ brain/awx148

Jucker, M., and Walker, L. C. (2013). Self-propagation of pathogenic protein aggregates in neurodegenerative diseases. Nature 501, 45-51. doi: 10.1038/ nature 12481

Kalliolia, E., Silajdžić, E., Nambron, R., Hill, N. R., Doshi, A., Frost, C., et al. (2014). Plasma melatonin is reduced in Huntington's disease. Mov. Disord. 29, 1511-1515.

La Morgia, C., Ross-Cisneros, F. N., Sadun, A. A., and Carelli, V. (2017). Retinal ganglion cells and circadian rhythms in alzheimer's disease, Parkinson's disease, and beyond. Front. Neurol. 8:162. doi: 10.3389/fneur.2017.00162

Lajoie, A. C., Lafontaine, A. L., Kimoff, R. J., and Kaminska, M. (2020). Obstructive sleep apnea in neurodegenerative disorders: current evidence in support of benefit from sleep apnea treatment. J. Clin. Med. 9:97.

Lazar, A. S., Panin, F., and Goodman, A. O. (2015). Sleep deficits but no metabolic deficits in premanifest Huntington's disease. Ann. Neurol. 78, 630-648. doi: 10.1002/ana.24495

Leng, Y., Blackwell, T., Cawthon, P. M., Ancoli-Israel, S., Stone, K. L., and Yaffe, K. (2020). Association of circadian abnormalities in older adults with an increased risk of developing Parkinson Disease. JAMA Neurol. 5:e201623.

Leng, Y., Musiek, E. S., Hu, K., Cappuccio, F. P., and Yaffe, K. (2019). Association between circadian rhythms and neurodegenerative diseases. Lancet Neurol. 18, 307-318. doi: 10.1016/s1474-4422(18)30461-7

Li, S., Wang, Y., Wang, F., Hu, L. F., and Liu, C. F. (2017). A new perspective for Parkinson's disease: circadian rhythm. Neurosci. Bull. 33, 62-72. doi: 10.1007/ s12264-016-0089-7

Li, P., Gao, L., Gaba, A., Yu, L., Cui, L., Fan, W., et al. (2020). Circadian disturbances in Alzheimer's disease progression: a prospective observational cohort study of community-based older adults. Lancet Healthy Longev. e96-e105. doi: 10.1016/ S2666-7568(20)30015-5

Liguori, C., Maestri, M., Spanetta, M., Placidi, F., Bonanni, E., Mercuri, N. B., et al. (2020). Sleep-disordered breathing and the risk of Alzheimer's disease. Sleep Med. Rev. 55:101375.

Liu, R. Y., Zhou, J. N., Hoogendijk, W. J., van Heerikhuize, J., Kamphorst, W., Unmehopa, U. A., et al. (2000). Decreased vasopressin gene expression in the biological clock of Alzheimer disease patients with and without depression. J. Neuropathol. Exp. Neurol. 59, 314-322. doi: 10.1093/jnen/59.4.314

Loddo, G., Calandra-Buonaura, G., and Sambati, L. (2017). The treatment of sleep disorders in parkinson's disease: from research to clinical practice. Front. Neurol. 8:42. doi: 10.3389/fneur.2017.00042

Long, J. M., and Holtzman, D. M. (2019). Alzheimer disease: an update on pathobiology and treatment strategies. Cell 179, 312-339. doi: 10.1016/j.cell. 2019.09.001

Lou, F., Li, M., Luo, X., and Ren, Y. (2018). CLOCK 3111T/C variant correlates with motor fluctuation and sleep disorders in chinese patients with Parkinson's Disease. Parkinsons. Dis. 4:4670380.

Lucey, B. P., McCullough, A., Landsness, E. C., Toedebusch, C. D., McLeland, J. S., Zaza, A. M., et al. (2019). Reduced non-rapid eye movement sleep is associated with tau pathology in early Alzheimer's disease. Sci. Transl. Med. 11:eaau6550. doi: 10.1126/scitranslmed.aau6550

Lysen, T. S., Darweesh, S. K. L., Ikram, M. K., Luik, A. I., and Ikram, M. A. (2019). Sleep and risk of parkinsonism and Parkinson's disease: a population-based study. Brain 142, 2013-2022. doi: 10.1093/brain/awz113

Mander, B. A., Winer, J. R., and Walker, M. P. (2017). Sleep and human aging. Neuron 94, 19-36.

Mao, W., Zhao, C., Ding, H., Liang, K., Xue, J., Chan, P., et al. (2018). Pyrosequencing analysis of methylation levels of clock genes in leukocytes from Parkinson's disease patients. Neurosci. Lett. 668, 115-119. doi: 10.1016/j.neulet. 2018.01.027

Masters, C. L., Bateman, R., Blennow, K., Rowe, C. C., Sperling, R. A., and Cummings, J. L. (2015). Alzheimer's disease. Nat. Rev. Dis. Primers 15:15056.

Mattis, J., and Sehgal, A. (2016). Circadian rhythms, sleep, and disorders of aging. Trends Endocrinol. Metab. 27, 192-203. doi: 10.1016/j.tem.2016.02.0 03

McCleery, J., Cohen, D. A., and Sharpley, A. L. (2014). Pharmacotherapies for sleep disturbances in Alzheimer's disease. Cochrane Database Syst. Rev. 21:CD009178.

Mueller, S. M., Petersen, J. A., and Jung, H. H. (2019). Exercise in Huntington's disease: current state and clinical significance. Tremor. Other Hyperkinet Mov. 9:601. doi: 10.5334/tohm.515

Musiek, E. S., Bhimasani, M., Zangrilli, M. A., Morris, J. C., Holtzman, D. M., and Ju, Y. S. (2018). Circadian rest-activity pattern changes in aging and preclinical alzheimer disease. Jama Neurol. 75, 582-590. doi: 10.1001/jamaneurol.2017. 4719

Musiek, E. S., and Holtzman, D. M. (2016). Mechanisms linking circadian clocks, sleep, and neurodegeneration. Science 354, 1004-1008. doi: 10.1126/science. aah4968

Nedergaard, M., and Goldman, S. A. (2020). Glymphatic failure as a final common pathway to dementia. Science 370, 50-56. doi: 10.1126/science.abb8739

Ortiz-Tudela, E., Martinez-Nicolas, A., and Diaz-Mardomingo, C. (2014). The characterization of biological rhythms in mild cognitive impairment. Biomed. Res. Int. 2014:524971.

Ouslander, J. G., Buxton, W. G., Al-Samarrai, N. R., Cruise, P. A., Alessi, C., and Schnelle, J. F. (1998). Nighttime urinary incontinence and sleep disruption among nursing home residents. J. Am. Geriatr. Soc. 46, 463-466. doi: 10.1111/ j.1532-5415.1998.tb02467.x

Pase, M. P., Himali, J. J., and Grima, N. A. (2017). Sleep architecture and the risk of incident dementia in the community. Neurology 89, 1244-1250. doi: 10.1212/wnl.0000000000004373

Patke, A., Young, M. W., and Axelrod, S. (2020). Molecular mechanisms and physiological importance of circadian rhythms. Nat. Rev. Mol. Cell Biol. 21, 67-84. doi: 10.1038/s41580-019-0179-2

Petit, D., Gagnon, J. F., Fantini, M. L., Ferini-Strambi, L., and Montplaisir, J. (2004). Sleep and quantitative EEG in neurodegenerative disorders. J. Psychosom. Res. 56, 487-496. doi: 10.1016/j.jpsychores.2004.02.001

Poewe, W., Seppi, K., Tanner, C. M., Halliday, G. M., Brundin, P., Volkmann, J., et al. (2017). Parkinson disease. Nat. Rev. Dis. Primers 23:17013. 
Rasmussen, M. K., Mestre, H., and Nedergaard, M. (2018). The glymphatic pathway in neurological disorders. Lancet Neurol. 17, 1016-1024. doi: 10.1016/ s1474-4422(18)30318-1

Raupach, A. K., Ehgoetz Martens, K. A., Memarian, N., Zhong, G., Matar, E., Halliday, G. M., et al. (2019). Assessing the role of nocturnal core body temperature dysregulation as a biomarker of neurodegeneration. J. Sleep Res. 11:e12939.

Saper, C. B., Scammell, T. E., and Lu, J. (2005). Hypothalamic regulation of sleep and circadian rhythms. Nature 437, 1257-1263. doi: 10.1038/nature04284

Seppi, K., Ray Chaudhuri, K., and Coelho, M. (2019). Update on treatments for nonmotor symptoms of Parkinson's disease-an evidence-based medicine review. Mov Disord. 34, 180-198. doi: 10.1002/mds.27602

Sharma, A., Lee, S., Kim, H., Yoon, H., Ha, S., and Kang, S. U. (2020). Molecular crosstalk between circadian rhythmicity and the development of neurodegenerative disorders. Front. Neurosci. 14:844. doi: 10.3389/fnins.2020. 00844

Shi, L., Chen, S. J., and Ma, M. Y. (2018). Sleep disturbances increase the risk of dementia: a systematic review and meta-analysis. Sleep Med. Rev. 40, 4-16. doi: $10.1016 /$ j.smrv.2017.06.010

Sixel-Doring, F., Zimmermann, J., Wegener, A., Mollenhauer, B., and Trenkwalder, C. (2016). The evolution of REM sleep behavior disorder in early Parkinson Disease. Sleep 39, 1737-1742. doi: 10.5665/sleep.6102

Skene, D. J., and Swaab, D. F. (2003). Melatonin rhythmicity: effect of age and Alzheimer's disease. Exp. Gerontol. 38, 199-206. doi: 10.1016/s0531-5565(02) 00198-5

Tandberg, E., Larsen, J. P., and Karlsen, K. (1999). Excessive daytime sleepiness and sleep benefit in Parkinson's disease: a community-based study. Mov Disord. 14, 922-927. doi: 10.1002/1531-8257(199911)14:6<922::aid-mds1003>3.0.co;2-7

Terum, T. M., Andersen, J. R., Rongve, A., Aarsland, D., Svendsboe, E. J., and Testad, I. (2017). The relationship of specific items on the neuropsychiatric inventory to caregiver burden in dementia: a systematic review. Int. J. Geriatr Psychiatry 32, 703-717. doi: 10.1002/gps.4704

van Wamelen, D. J., Aziz, N. A., Anink, J. J., van Steenhoven, R., Angeloni, D., Fraschini, F., et al. (2013). Suprachiasmatic nucleus neuropeptide expression in patients with Huntington's Disease. Sleep 36, 117-125.

Vetrano, D. L., Pisciotta, M. S., Lo Monaco, M. R., Onder, G., Laudisio, A., Brandi, V., et al. (2015). Association of depressive symptoms with circadian blood pressure alterations in Parkinson's disease. J. Neurol. 262, 2564-2571. doi: 10.1007/s00415-015-7887-0

Videnovic, A., and Golombek, D. (2013). Circadian and sleep disorders in Parkinson's disease. Exp. Neurol. 243, 45-56.

Videnovic, A., and Golombek, D. (2017). Circadian dysregulation in Parkinson's disease. Neurobiol. Sleep Circadian Rhythms 2, 53-58. doi: 10.1016/j.nbscr. 2016.11.001

Videnovic, A., Lazar, A. S., Barker, R. A., and Overeem, S. (2014a). 'The clocks that time us'- circadian rhythms in neurodegenerative disorders. Nat. Rev. Neurol. 10, 683-693. doi: 10.1038/nrneurol.2014.206

Videnovic, A., Noble, C., Reid, K. J., Peng, J., Turek, F. W., Marconi, A., et al. (2014b). Circadian melatonin rhythm and excessive daytime sleepiness in Parkinson disease. JAMA Neurol. 71, 463-469. doi: 10.1001/jamaneurol.2013. 6239

Videnovic, A., Leurgans, S., Fan, W., Jaglin, J., and Shannon, K. M. (2009). Daytime somnolence and nocturnal sleep disturbances in Huntington disease. Parkinsonism Relat. Disord. 15, 471-474. doi: 10.1016/j.parkreldis.2008.10.002

Videnovic, A., and Willis, G. L. (2016). Circadian system - a novel diagnostic and therapeutic target in Parkinson's disease? Mov. Disord. 31, 260-269. doi: $10.1002 /$ mds. 26509
Virta, J. J., Heikkila, K., and Perola, M. (2013). Midlife sleep characteristics associated with late life cognitive function. Sleep 36, 1533-1541, 1541A.

Vitiello, M. V., Prinz, P. N., Williams, D. E., Frommlet, M. S., and Ries, R. K. (1990). Sleep disturbances in patients with mild-stage Alzheimer's disease. J. Gerontol. 45, M131-M138.

Wang, C., and Holtzman, D. M. (2020). Bidirectional relationship between sleep and Alzheimer's disease: role of amyloid, tau, and other factors. Neuropsychopharmacology $\quad 45, \quad 104-120$. doi: 10.1038/s41386-0190478-5

Wang, J. L., Lim, A. S., and Chiang, W. Y. (2015). Suprachiasmatic neuron numbers and rest-activity circadian rhythms in older humans. Ann. Neurol. 78, 317-322. doi: 10.1002/ana.24432

Weissova, K., Bartos, A., Sladek, M., Novakova, M., and Sumova, A. (2016). Moderate changes in the circadian system of alzheimer's disease patients detected in their home environment. PLoS One 11:e146200. doi: 10.1371/ journal.pone. 0146200

Wiegand, M., Moller, A. A., and Lauer, C. J. (1991). Nocturnal sleep in Huntington's disease. J. Neurol. 238, 203-208.

Winer, J. R., Mander, B. A., Kumar, S., Reed, M., Baker, S. L., Jagust, W. J., et al. (2020). Sleep disturbance forecasts $\beta$-Amyloid accumulation across subsequent years. Curr. Biol. 30, 4291.e3-4298.e3.

Wu, Y. H., Fischer, D. F., Kalsbeek, A., Garidou-Boof, M. L., van der Vliet, J., van Heijningen, C., et al. (2006). Pineal clock gene oscillation is disturbed in Alzheimer's disease, due to functional disconnection from the "master clock". FASEB J. 20, 1874-1876. doi: 10.1096/fj.05-4446fje

Wu, Y. H., Zhou, J. N., Van Heerikhuize, J., Jockers, R., and Swaab, D. F. (2007). Decreased MT1 melatonin receptor expression in the suprachiasmatic nucleus in aging and Alzheimer's disease. Neurobiol. Aging 28, 1239-1247. doi: 10.1016/ j.neurobiolaging.2006.06.002

Xie, L., Kang, H., Xu, Q., Chen, M. J., Liao, Y., Thiyagarajan, M., et al. (2013). Sleep drives metabolite clearance from the adult brain. Science 342, 373-377. doi: $10.1126 /$ science. 1241224

Yong, M. H., Fook-Chong, S., Pavanni, R., Lim, L. L., and Tan, E. K. (2011). Case control polysomnographic studies of sleep disorders in Parkinson's disease. PLoS One 6:e22511. doi: 10.1371/journal.pone.0022511

You, S., Jeon, S. M., Do, S. Y., and Cho, Y. W. (2019). Restless legs syndrome in Parkinson's disease patients: clinical features including motor and nonmotor symptoms. J. Clin. Neurol. 15, 321-327. doi: 10.3988/jcn.2019.15. 3.321

Zhang, Y., Ren, R., Yang, L., Zhou, J., Li, Y., Shi, J., et al. (2019). Sleep in Huntington's disease: a systematic review and meta-analysis of polysomongraphic findings. Sleep 42 , zsz154.

Zhou, J. N., Hofman, M. A., and Swaab, D. F. (1995). VIP neurons in the human SCN in relation to sex, age, and Alzheimer's disease. Neurobiol. Aging 16, 571-576. doi: 10.1016/0197-4580(95)00043-e

Conflict of Interest: The authors declare that the research was conducted in the absence of any commercial or financial relationships that could be construed as a potential conflict of interest.

Copyright (C) 2021 Fifel and Videnovic. This is an open-access article distributed under the terms of the Creative Commons Attribution License (CC BY). The use, distribution or reproduction in other forums is permitted, provided the original author(s) and the copyright owner(s) are credited and that the original publication in this journal is cited, in accordance with accepted academic practice. No use, distribution or reproduction is permitted which does not comply with these terms. 